\title{
SOX4 cooperates with neurogenin 3 to regulate endocrine pancreas formation in mouse models
}

\author{
Eric E. Xu • Nicole A. J. Krentz • Sara Tan • \\ Sam Z. Chow $\cdot$ Mei Tang • Cuilan Nian • Francis C. Lynn
}

Received: 23 August 2014 / Accepted: 9 January 2015 / Published online: 5 February 2015

(C) Springer-Verlag Berlin Heidelberg 2015

\begin{abstract}
Aims/hypothesis The sex-determining region Y (SRY)-related high mobility group (HMG) box (SOX) family of transcription factors is essential for normal organismal development. Despite the longstanding knowledge that many SOX family members are expressed during pancreas development, a role for many of these factors in the establishment of insulinproducing beta cell fate remains to be determined. The aim of this study is to elucidate the role of SOX4 during beta cell development.

Methods We used pancreas and endocrine progenitor mouse knockouts of Sox4 to uncover the roles of SOX4 during pancreas development. Lineage tracing and in vitro models were used to determine how SOX4 regulates beta cell formation and understand the fate of Sox4-null endocrine lineage cells. Results This study demonstrates a progenitor cellautonomous role for SOX4 in regulating the genesis of beta cells and shows that it is required at multiple stages of the process. SOX4 deletion in the multipotent pancreatic progenitors resulted in impaired endocrine progenitor cell
\end{abstract}

Electronic supplementary material The online version of this article (doi:10.1007/s00125-015-3507-x) contains peer-reviewed but unedited supplementary material, which is available to authorised users.

E. E. Xu • N. A. J. Krentz $\cdot$ S. Tan $\cdot$ S. Z. Chow $\cdot$ M. Tang $\cdot$ C. Nian $\cdot$

F. C. Lynn $(\triangle)$

Diabetes Research Program, Child and Family Research Institute,

A4-184, 950 West 28 Ave, Vancouver, BC V5Z 4H4, Canada

e-mail: francis.lynn@ubc.ca

E. E. Xu • N. A. J. Krentz $\cdot$ M. Tang $\cdot$ C. Nian $\cdot$ F. C. Lynn

Department of Surgery, University of British Columbia,

Vancouver, BC, Canada

F. C. Lynn

Department of Cellular and Physiological Sciences, University of

British Columbia, Vancouver, BC, Canada differentiation. Deletion of SOX4 later in the Neurog3-expressing cells also caused reductions in beta cells. Lineage studies showed loss of Sox4 in endocrine progenitors resulted in a block in terminal islet cell differentiation that was attributed to reduction in the production of key beta cell specification factors.

Conclusions/interpretation These results demonstrate that SOX4 is essential for normal endocrine pancreas development both concomitant with, and downstream of, the endocrine fate decision. In conclusion, these studies position Sox4 temporally in the endocrine differentiation programme and provide a new target for improving in vitro differentiation of glucoseresponsive pancreatic beta cells.

Keywords Basic science $\cdot$ Cell lines · Human · Islet development · Islet transplantation · Islets $\cdot$ Knockout mice Transcription factors

\begin{tabular}{|c|c|}
\hline \multicolumn{2}{|c|}{ Abbreviations } \\
\hline+ & Hormone-immunoreactive (cells) \\
\hline CHGA & Chromogranin A \\
\hline ChIP & Chromatin immunoprecipitation \\
\hline Cre & Cre recombinase \\
\hline $\mathrm{E}$ & Embryonic day \\
\hline EMS4KO & Neurog3-Cre mT/mG Sox $4^{\text {flox/flox }}$ \\
\hline ES4KO & Neurog3-Cre Sox $4^{\text {flox/flox }}$ \\
\hline GCG & Glucagon \\
\hline GF & Green fluorescence \\
\hline (e)GFP & (enhanced) Green fluorescent protein \\
\hline GHRL & Ghrelin \\
\hline HA & Haemagglutinin \\
\hline hESC & Human embryonic stem cell \\
\hline $\mathrm{HMG}$ & High mobility group \\
\hline INS & Insulin \\
\hline Mafa & $\begin{array}{l}\text { v-maf Avian musculoaponeurotic fibrosarcoma } \\
\text { oncogene homologue A }\end{array}$ \\
\hline
\end{tabular}




\begin{tabular}{|c|c|}
\hline $\begin{array}{l}\mathrm{mPAC} \\
\mathrm{mT} / \mathrm{mG}\end{array}$ & $\begin{array}{l}\text { Mouse pancreatic ductal adenocarcinoma } \\
\text { Membrane-targeted tdTomato/membrane- } \\
\text { targeted enhanced GFP }\end{array}$ \\
\hline NEUROD1 & Neurogenic differentiation 1 \\
\hline NEUROG3 & Neurogenin 3 \\
\hline NKX2.2 & NK2 homeobox 2 \\
\hline NKX6.1 & NK6 homeobox 1 \\
\hline ONECUT1 & One cut homeobox 1 \\
\hline PAX4 & Paired box 4 \\
\hline PDX1 & Pancreatic and duodenal homeobox 1 \\
\hline PPY & Pancreatic polypeptide \\
\hline PS4KO & $P d x 1$-Cre $\operatorname{Sox} 4^{\text {flox/flox }}$ \\
\hline PTF1A & Pancreas transcription factor 1 subunit $\alpha$ \\
\hline $\mathrm{q}(\mathrm{PCR})$ & Quantitative (PCR) \\
\hline $\mathrm{RF}$ & Red fluorescence \\
\hline RFP & Red fluorescent protein \\
\hline RFX6 & Regulatory factor X, 6 \\
\hline SOX & SRY-related high mobility group box \\
\hline SRY & Sex-determining region $\mathrm{Y}$ \\
\hline SST & Somatostatin \\
\hline WT & Wild type \\
\hline
\end{tabular}

\section{Introduction}

In mice, pancreas development begins when pancreatic and duodenal homeobox 1 (PDX1), pancreas transcription factor 1 subunit $\alpha$ (PTF1A) and sex-determining region Y (SRY)-related high mobility group (HMG) box (SOX)9-expressing epithelial cells bud from the posterior foregut endoderm at embryonic day (E) 9 [1-3]. Signals from adjacent tissues support budding and as development proceeds, two waves of differentiation occur [4-8]. The primary transition, from E8.5-10.5, is marked by low numbers of hormone-immunoreactive $\left(^{+}\right)$cells; the secondary transition, from E12.5-15.5, is denoted by a large wave of exocrine and endocrine cell differentiation [9]. The restriction towards a terminally differentiated endocrine cell occurs progressively [10].

Endocrine differentiation requires inhibition of $\mathrm{NOTCH}$ signalling and transient activation of neurogenin 3 (NEUROG3) in epithelial trunk progenitor cells, and is accompanied by exit from the cell cycle and migration into the mesenchyme [11-18]. Downstream, regulators of beta cell specification include: paired box 4 (PAX4), neurogenic differentiation 1 (NEUROD1), regulatory factor X, 6 (RFX6), NK2 homeobox 2 (NKX2.2), NK6 homeobox 1 (NKX6.1) and $\mathrm{V}$-maf avian musculoaponeurotic fibrosarcoma oncogene homologue A (MAFA), among others [19-25]. While a number of these factors are essential for normal beta cell development, it is unclear if any transcription factor couples the endocrine progenitor fate choice with downstream terminal differentiation.
Here, we demonstrate that SOX4 may play this bridging role during endocrine cell genesis.

SOX proteins are an evolutionarily ancient family of complex-forming transcriptional activators and repressors that are identified by a conserved HMG DNA-binding domain $[26,27]$. A plethora of SOX genes has been demonstrated to be expressed within the developing pancreas, including most of the C, D, E, F, G and H subfamily members [28]. Early during pancreas formation, expression of an E group family member, SOX9, is critical for the maintenance of pancreatic progenitor identity [1].

Previous work has demonstrated SOX4 expression in the embryonic human, mouse and zebrafish pancreas [28-30]. Null mutation of Sox4 results in cardiac abnormalities, defects in blood flow and embryonic lethality by E14.5 prior to completion of the bulk of endocrine cell differentiation. However, significant reductions of insulin (INS) and/or glucagon (GCG) expression were observed by us and others [29, 31].

This study expands on these observations to define the spatiotemporal importance of SOX4 during endocrine formation in vivo. SOX4 is demonstrated to be required in a cellautonomous capacity for efficient endocrine cell differentiation both prior to and downstream of NEUROG3. As such, the function of SOX4 during pancreatic endocrine cell formation mirrors roles of SOX family genes in other systems: to bridge key developmental transitions and couple fate decisions.

\section{Methods}

Chemicals and reagents Chemicals were purchased from Sigma Aldrich (Oakville, ON, Canada) or Fisher Scientific (Ottawa, ON, Canada). The antibodies used are listed in electronic supplementary material (ESM) Table 1. Oligonucleotides were purchased from IDT (Coralville, IA, USA; ESM Table 2). Tissue culture reagents were from Hyclone (Logan, UT, USA) and cultureware from BD-Falcon (Fisher Scientific).

Tissues The University of British Columbia (UBC) Animal Care Committee approved all mouse breeding and experiments, with E0.5 set as noon after discovery of a vaginal plug. Mouse strains included membrane-targeted tdTomato/ membrane-targeted enhanced (e) green fluorescent protein (GFP; mT/mG) [32], Neurog3-Cre recombinase (Cre) [33] (Jackson Laboratory, Bar Harbor, ME, USA), Pdxl-Cre [17] (Mutant Mouse Regional Resource Centers [MMRRC], Davis, CA, USA) and Sox $4^{\text {flox }}$ [34]. Pdxl-Cre Sox $4^{\text {flox/flox }}$ (PS4KO) and Neurog3-Cre Sox $4^{\text {flox/flox }}$ (ES4KO) controls were Cre negative and Sox $4^{\text {flox/WT }}$ or Sox $4^{\text {flox/flox }}$ littermates (where WT is wild type). Neurog3-Cre $\mathrm{mT} / \mathrm{mG}$ Sox 4 flox/flox (EMS4KO) controls were: Neurog3-Cre $\mathrm{mT} / \mathrm{mG}^{\text {flox/WT }}$ or $\mathrm{mT} / \mathrm{mG}^{\text {flox/flox }}$ and $S o x^{\text {flox/WT }}$ double heterozygotes. The 
University of Alberta Research and Transplant Islet Isolation Cores obtained human islets under informed consent.

Tissue harvest and preparation Tissue was harvested and fixed in $4 \%$ (wt/vol.) paraformaldehyde in PBS $\left(4^{\circ} \mathrm{C}\right)$. Tissue samples were then dehydrated and paraffin embedded or frozen as described [35]. Sections, $5 \mu \mathrm{m}$ paraffin or $10 \mu \mathrm{m}$ frozen, were rehydrated as necessary prior to antigen retrieval.

Immunostaining and imaging Please see ESM for antibodies, in situ hybridisation, TUNEL and 5-ethynyl-2'-deoxyuridine (EdU) staining methods. Antigen retrieval was performed in citrate buffer ( $\mathrm{pH} 6$ at $95^{\circ} \mathrm{C}$ for $20 \mathrm{~min}$ ). Slides were blocked for $1 \mathrm{~h}$ each in $5 \%$ (vol./vol.) horse serum followed by donkey anti-mouse $\mathrm{IgG}$. Sections were incubated overnight at $4{ }^{\circ} \mathrm{C}$ with primary antibody and for $1 \mathrm{~h}$ at $20^{\circ} \mathrm{C}$ with secondary antibodies plus nuclear stains. Tyramide signal amplification (TSA) was carried out for detection of SOX4 (Perkin Elmer, Woodbridge, ON, Canada). Imaging was carried out with sequential scanning on a Leica SP8 confocal imaging system (Concord, ON, Canada).

Morphometric analyses and quantification Serial sections were chosen at 100 or $50 \mu \mathrm{m}$ intervals for E18.5 or E15.5 embryos, respectively. The whole section was imaged and tiled and immunopositive cells were counted using CellProfiler (Cambridge, MA, USA) [36]. Counts were normalised to total pancreatic nuclei using CellProfiler or the sum of pancreatic area calculated using Image-Pro Analyzer (Media Cybernetics, Rockville, MD, USA).

Adenoviral generation, infection and cell culture Sox4 adenovirus was generated using the pAdTrack system (Addgene, Cambridge, MA, USA) [37]. Viruses were purified with the Adeno-X Maxi adenovirus purification system (Clontech, Mountain View, CA, USA). Please see ESM Methods for gene targeting, human embryonic stem cells (hESCs) and FACS methods. Mouse pancreatic ductal adenocarcinoma (mPACs) cells were infected at a multiplicity of infection (MOI) 100:1 for $2 \mathrm{~h}$. Cells were washed briefly in PBS, media was replaced and they were cultured for $48 \mathrm{~h}$.

Real-time PCR RNA was isolated with TRIzol and DNAse treated and cDNA was synthesised using Superscript II reverse transcriptase (Life Technologies, Burlington, ON, Canada). A $40 \mathrm{ng}$ sample of cDNA was used as template for quantitative (q)PCR carried out in a ViiA7 qPCR machine (Life Technologies). Expression was determined using the $\Delta \Delta \mathrm{C}_{\mathrm{t}}$ method with glucouronidase B (Gusb) as a reference gene [35].

Luciferase assay mPACL20 cells were seeded $\left(2 \times 10^{5}\right.$ cells) in 24-well plates and transfected using Lipofectamine 2000
(Life Technologies) with $1 \mu \mathrm{g}$ reporter, $50 \mathrm{ng}$ cytomegalovirus promoter (pCMV)-renilla luciferase and $100 \mathrm{ng}$ expression vectors, balanced with empty expression vector. Cells were incubated for $48 \mathrm{~h}$ prior to determination of luciferase activity using the Dual-Luciferase Reporter (DLR) Assay (Promega, Madison, WI, USA) on a SpectraMaxL luminometer (Molecular Devices, Sunnyvale, CA, USA).

Chromatin immunoprecipitation Chromatin immunoprecipitation (ChIP) was performed as previously described [35]. The only change being that ChIP was carried out in the presence of $160 \mathrm{mmol} / \mathrm{l} \mathrm{NaCl}, 0.25 \%$ (wt/vol.) deoxycholate and $0.025 \%$ (vol./vol.) Triton X-100.

Statistical analysis All statistical analyses were performed with Prism5 (GraphPad Software, La Jolla, CA, USA). Two-tailed unpaired Student's $t$ tests or one-way ANOVA with Tukey post-hoc tests were performed, with $p \leq 0.05$ considered significant. Error bars represent SEM.

\section{Results}

SOX4 is dynamically expressed during mouse pancreas formation As seen in Fig. 1a, Sox4 is expressed throughout pancreas development, with the highest levels at E11.5. A gradual decline in Sox 4 message was observed; however, significant expression was observed during the secondary transition when the majority of NEUROG3 ${ }^{+}$endocrine progenitors are derived (E12.5-14; Fig. 1a). During human embryonic stem cell differentiation $\mathrm{SOX} 4$ is expressed at high levels in stage 3 and 4 pancreatic progenitors (Fig. 1b).

Because suitable antibodies were not initially available, in situ hybridisation was carried out to localise Sox4 expression (ESM Fig. 1). The epithelium at this stage was cord-like with $\mathrm{GCG}^{+}$cells found in proximity to the trunk (ESM Fig. 1a). Low expression of Sox 4 mRNA was observed throughout the epithelium (E14.5; ESM Fig. 1b). Occasional cells expressing higher levels of Sox 4 were noted scattered through the epithelium (arrowheads ESM Fig. 1b): an expression pattern reminiscent of the bipotent trunk cells that activate NEUROG3 $[13,38]$. Next, immunostaining was used to determine the spatiotemporal distribution of SOX4. Early during pancreatic organogenesis (E11.5 and E12.5), SOX4 co-localised with PDX1 and NKX6.1 in the multipotent progenitors and trunk cells respectively (Fig. 1c, d; ESM Fig. 2b) [39]. Following the secondary transition, SOX4 was progressively excluded from pancreatic epithelial cells; however, it remained highly expressed in all NEUROG3-expressing endocrine progenitors (Fig. 1e, f) and in nascent endocrine cells (ESM Fig. 2b). By E17.5, SOX4 expression was confined largely to endocrine cells (ESM Fig. 2d). 

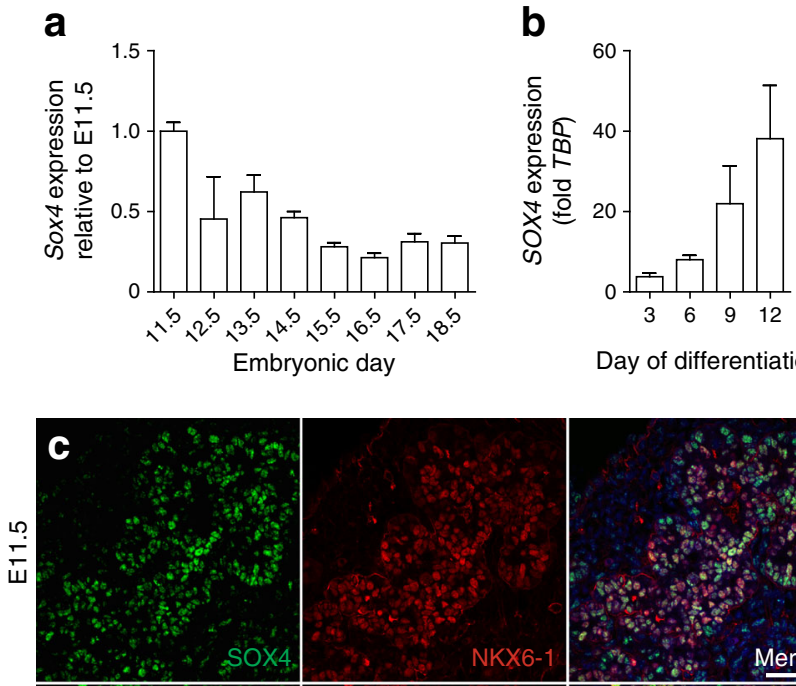

Day of differentiation
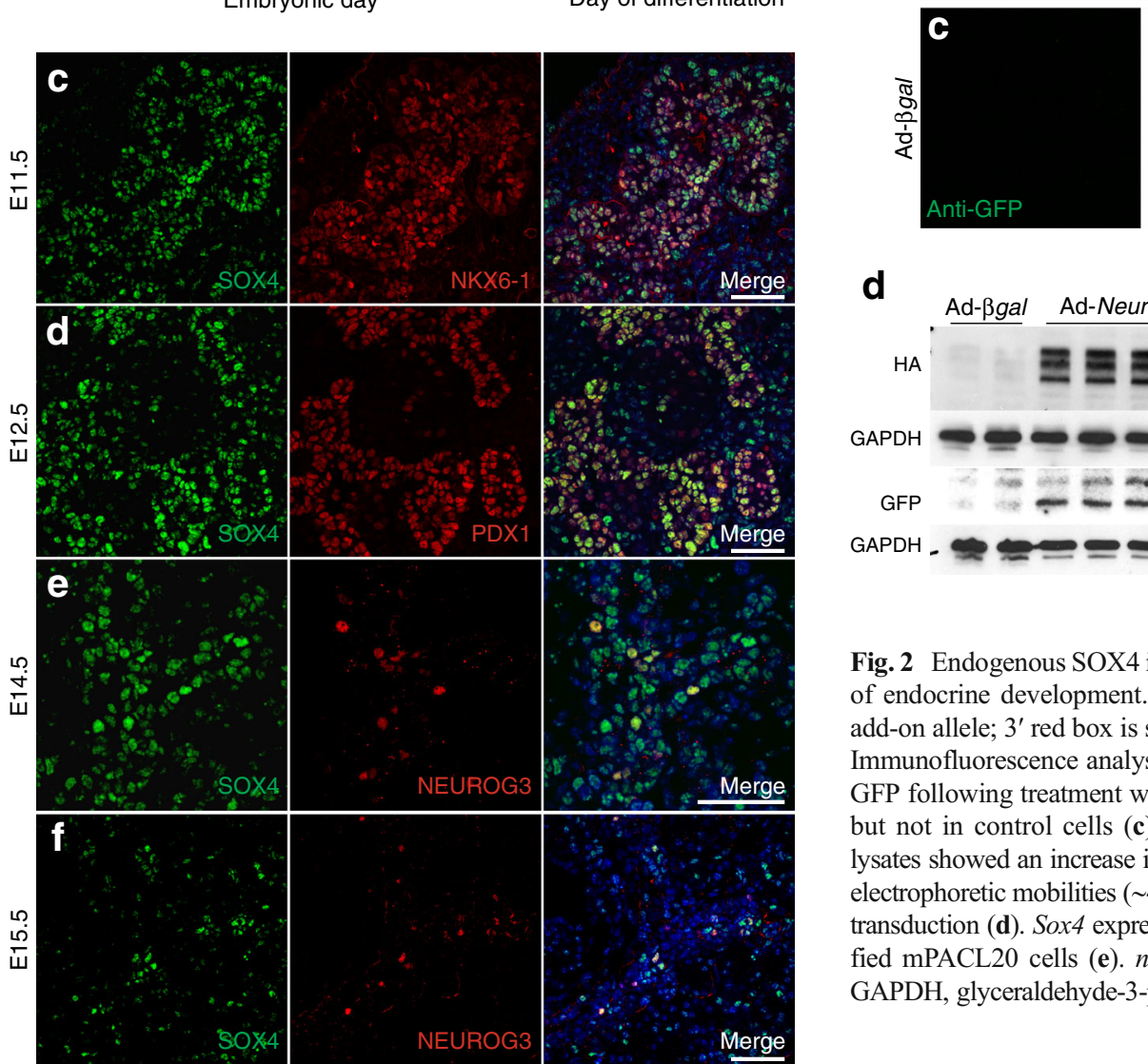

Fig. 1 SOX4 is expressed during pancreas development and becomes restricted to endocrine cells. qPCR analyses of Sox 4 expression in E11.5 to E18.5 mouse pancreas (a) and following directed differentiation of hESCs (b). Coimmunostaining of SOX4 (green) (c-f) with: (c) NKX61 (red), E11.5; (d) PDX1 (red), E12.5; and (e, f) NEUROG3 (red) (e) E14.5 and (f) E15.5. $n \geq 3$; scale bars, $50 \mu \mathrm{m}$. TBP, TATA-binding protein

To verify that SOX4 was expressed in NEUROG3 ${ }^{+}$endocrine progenitor cells, the mouse mPACL20 pancreatic ductal cell line, which has previously been used to model endocrine cell differentiation, was used [40, 41]. Using transcription activator-like effector nuclease (TALEN)-driven homologous recombination in the mPACL20 cells, a haemagglutinin (HA) tag followed by 2A-GFP was knocked onto Sox4 (mPAC-Sox4-HA; Fig. 2a) and 11 clonal lines were derived with $23 \%$ targeting efficiency.

As predicted from the immunostaining results (Fig. 1c-f), when this clonal mPAC-Sox4-HA cell line was transduced

\section{d}

a

\begin{tabular}{l|ll|l} 
Targeted & Sox4 & HA-2A & eGFP \\
\hline
\end{tabular}
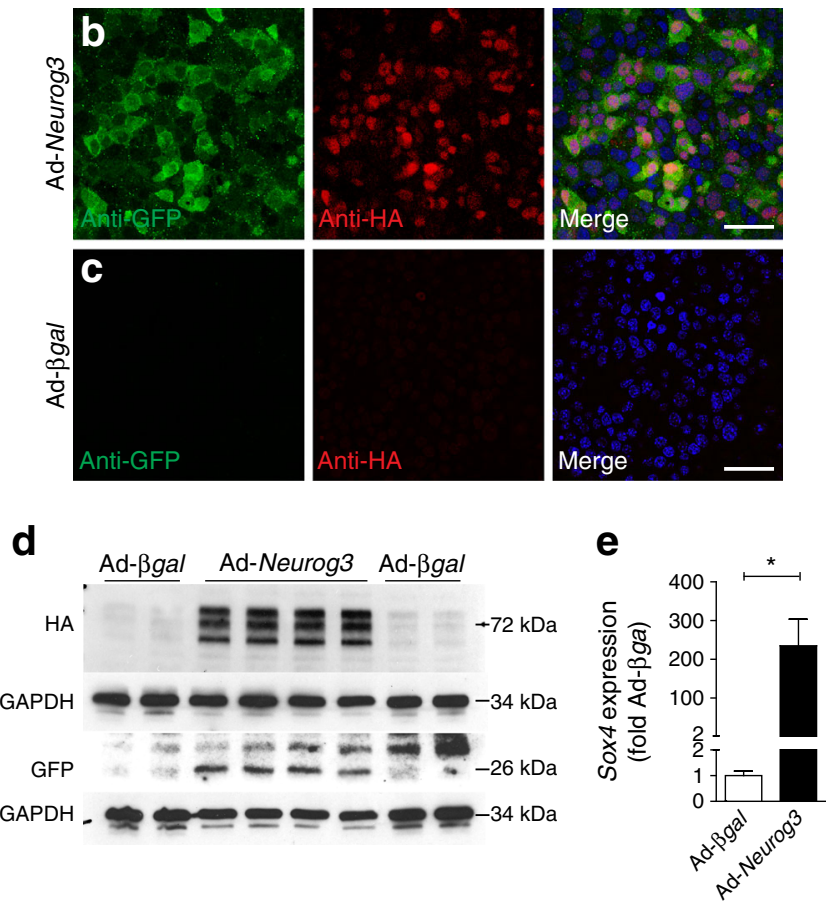

Fig. 2 Endogenous SOX4 is induced by NEUROG3 in the mPAC model of endocrine development. Schematic of Sox4-HA-2A-eGFP knock-in add-on allele; $3^{\prime}$ red box is stop codon (a; see ESM Methods for details). Immunofluorescence analyses show induction of HA-tagged SOX4 and GFP following treatment with Ad-Neurog3 in mPAC-Sox4-HA cells (b) but not in control cells (c). Western blotting of mPAC-Sox4-HA cell lysates showed an increase in HA and GFP immunoreactivity at predicted electrophoretic mobilities $\left(\sim 47 \mathrm{kDa}\right.$ and $\left.\sim 70 \mathrm{kDa}^{+}\right)$following Ad-Neurog3 transduction (d). Sox4 expression is increased by Ad-Neurog3 in unmodified mPACL20 cells (e). $n \geq 3 ;{ }^{*} p \leq 0.05$. $\beta g a l$ is also known as $b g a L$. GAPDH, glyceraldehyde-3-phosphate dehydrogenase

with a Neurog3 adenovirus (Ad-Neurog3) [41], marked increases in endogenous promoter activity led to appreciable HA and GFP that were detectable by both immunostaining (Fig. 2b, c) and western blotting (Fig. 2d). In support, unmodified mPACL20 cells transduced with Ad-Neurog3 showed a 240-fold increase in Sox4 message expression (Fig. 2e).

These observations show that SOX4 is widely produced in the multipotent pancreatic progenitors prior to endocrine differentiation and that its expression is maintained within the endocrine progenitors. In order to assess whether there is an important cell-autonomous role for SOX4 during pancreatic or endocrine cell formation, loss-of-function approaches were used.

SOX4 is necessary for endocrine progenitor cell formation To determine if SOX4 is important prior to endocrine progenitor formation, mice harbouring a floxed Sox 4 allele (Sox $4^{\text {flox }}$ ) were crossed with $P d x 1$-Cre transgenic mice to generate 
pancreatic Sox4-knockout embryos (PS4KO). Lineage analyses indicate that recombination is extremely efficient early during pancreas organogenesis $(>95 \%$ of epithelial cells at E12.5; ESM Fig. 2a). In agreement, SOX4 immunostaining at E15.5 verified that SOX4 protein level was significantly reduced compared with controls (ESM Fig. 2b, c).

Quantification of endocrine cell types at E18.5 revealed that pancreatic deletion of Sox4 significantly reduced $\mathrm{INS}^{+} \mathrm{GCG}^{+}$ (Fig. 3a, b, i, j), somatostatin ${ }^{+}$([SST $\left.{ }^{+}\right]$Fig. 3c, d, l), and pancreatic polypeptide ${ }^{+}\left(\left[\mathrm{PPY}^{+}\right]\right.$Fig. 3e, f, $\mathrm{m}$ ) cells, without changes in ghrelin $^{+}\left(\mathrm{GHRL}^{+}\right)$cells (Fig. 3c, d, k). To address the possibility of a defect in early endocrine cell differentiation, bipotent $\mathrm{SOX}^{+}$ cells and NEUROG3 ${ }^{+}$endocrine progenitor cells were quantified. A significant reduction in NEUROG3 ${ }^{+}$cells, but not $\mathrm{SOX9}^{+}$cells, was observed (Fig. $3 \mathrm{~g}, \mathrm{~h}, \mathrm{n}$, o); suggesting that SOX4 is dispensable until endocrine differentiation is initiated within the $\mathrm{SOX}^{+}$progenitor $[38,42]$. As expected, neither apoptosis (ESM Fig. 3e-h) nor proliferation (ESM Fig. 3c, d) accounted for the differences in NEUROG3 ${ }^{+}$ cells at E15.5.

Despite the marked phenotype and loss of SOX4 protein (ESM Fig. 2c), Sox4 message was significantly reduced but detectable in knockout pancreas by qPCR (Fig. 3p). This may result from a long half-life of Sox4 message or expression from neurons, blood vessels and mesenchyme that are closely associated with the epithelium. Expression of related SOXC class family members, Sox11 and Sox12 (Fig. 3q, r), was not significantly different, suggesting their upregulation did not compensate for loss of Sox4. In sum, these findings demonstrate SOX4 is essential for efficient endocrine progenitor formation.

SOX4 activates the Neurog 3 promoter and induces NEUROG 3 expression To investigate whether the cooperative actions of NEUROG3 and SOX4 are important for endocrine cell genesis, unmodified mPACL20 pancreatic ductal cells were transduced with Ad-Sox4 and Ad-Neurog3 (Fig. 4a) [41, 42]. Co-transduction of mPACL20 cells resulted in a significant increase in endogenous Neurog3 expression (Fig. 4a).

In order to determine if SOX4 binds and directly regulates NEUROG3 in the context of development, the modified mPAC-Sox4-HA cells were transduced with Ad-Neurog3 to induce endogenous Sox4 (Fig. 2e) and ChIP was carried out using anti-HA antibodies (Fig. 4b). Significant enrichment of SOX4-HA at a conserved region of the Neurog3 promoter containing two SOX4 consensus binding sites (ESM Fig. 4) was observed. Luciferase assays next demonstrated that SOX 4 can transactivate a $2 \mathrm{~kb}$ fragment of the Neurog3 promoter (Fig. 4c) [43]. Collectively, these results suggest that SOX4 expression within a subset of bipotent trunk progenitors enhances NEUROG3 expression and drives subsequent endocrine progenitor cell formation.

SOX4 regulates endocrine cell differentiation downstream of NEUROG3 SOX4 expression is maintained at high levels in both mouse and human islets (ESM Figs 2 and 5); however, NEUROG3 expression is not [44]. In order to test if the
Fig. 3 PS4KO mice have defects in endocrine cell genesis. Immunohistochemical analyses of hormone immunoreactive $\left(^{+}\right)$ cells in control $(\mathbf{a}, \mathbf{c}, \mathbf{e}, \mathbf{g})$ and PS4KO (b, d, f, h) pancreas: $(\mathbf{a}, \mathbf{b}) \mathrm{INS}^{+}$(green) and $\mathrm{GCG}^{+}$ (red), E18.5; (c, d) GHRL ${ }^{+}$ (green) and $\mathrm{SST}^{+}$(red) E18.5; (e, f) $\mathrm{PPY}^{+}$(green) and $\mathrm{INS}^{+}$ (red), E18.5; (g, h) NEUROG3 ${ }^{+}$ (green) and $\mathrm{SOX}^{+}$(red), E15.5. PS4KO (black bars) had significantly reduced numbers of $\mathrm{INS}^{+}(\mathbf{i}), \mathrm{GCG}^{+}(\mathbf{j}), \mathrm{SST}^{+}$(I), $\mathrm{PPY}^{+}$ $(\mathbf{m})$ and $\mathrm{NEUROG}^{+}(\mathbf{n})$ cells but not in $\mathrm{GHRL}^{+}(\mathbf{k})$ or $\mathrm{SOX9}^{+}(\mathbf{o})$ cells compared with controls (white bars). Sox4 expression (p) was significantly reduced at E15.5 in PS4KO pancreas without changes in Sox11 (q) or Sox12 (r). $n \geq 3 ;{ }^{*} p \leq 0.05$; scale bars, $50 \mu \mathrm{m}$; nuclei are stained blue
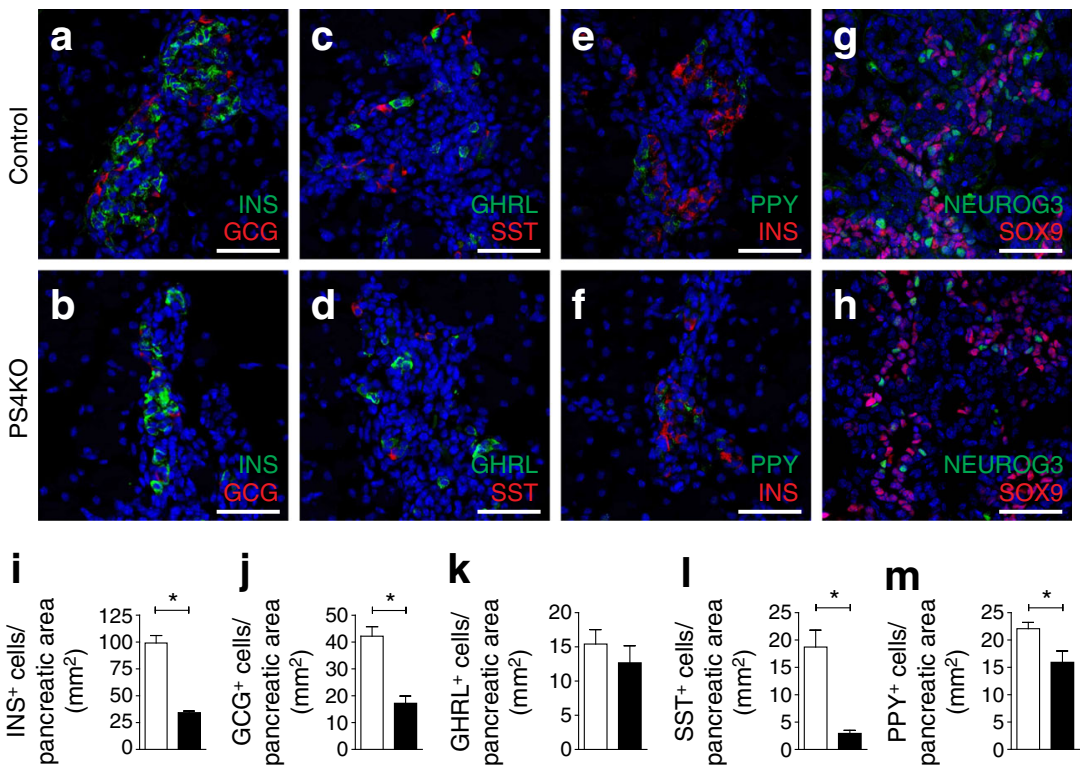

j $\quad k$
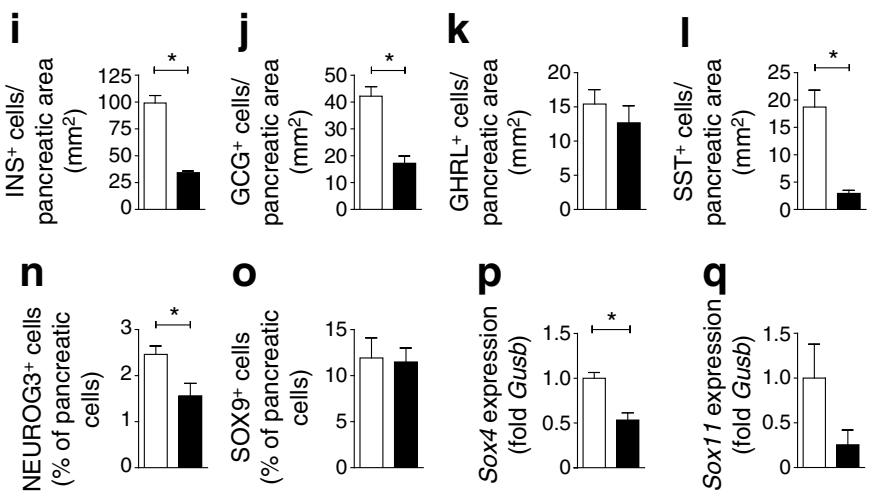

m
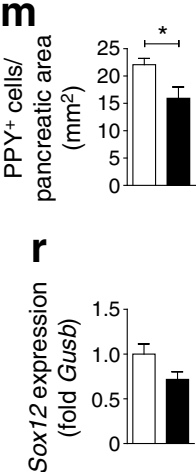

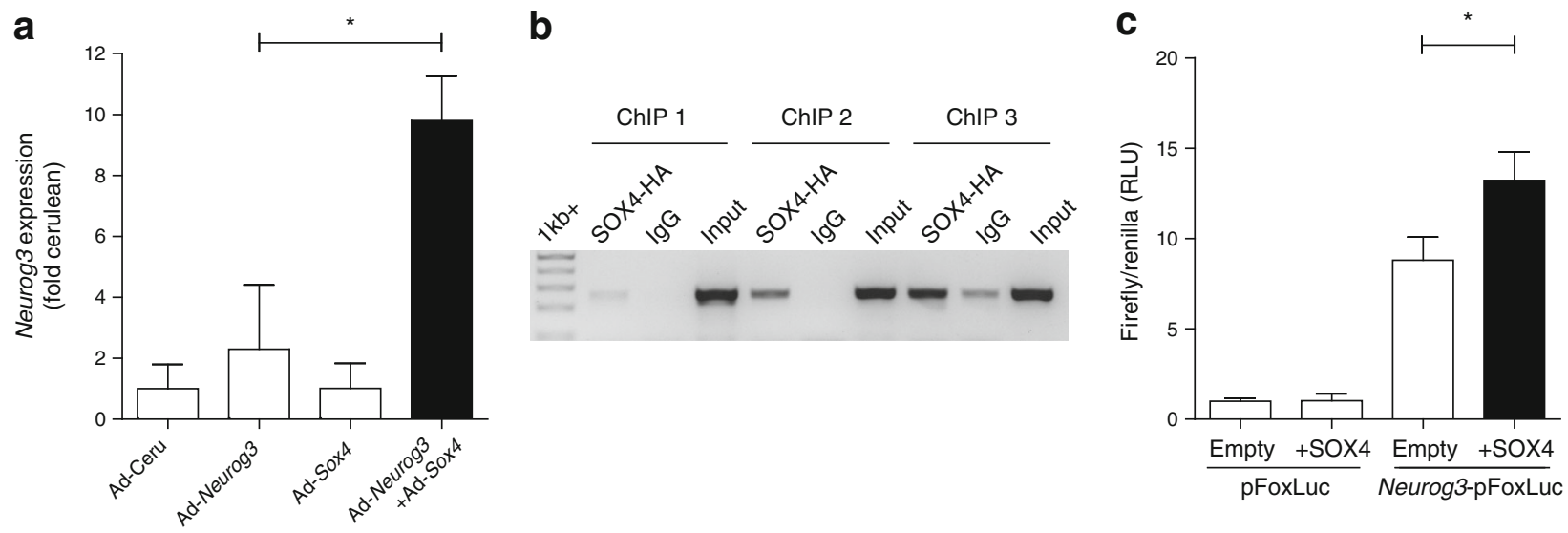

Fig. 4 SOX4 directly regulates Neurog3 expression in the mPAC model of endocrine development. Ad-Neurog3 and Ad-Sox4 cooperatively increase expression from the Neurog3 locus in mPACL20 cells (a). HA-ChIP (b) following Ad-Neurog3 showed HA-tagged SOX4 protein

maintenance of Sox4 expression downstream of Neurog3 remains important for islet cell formation, mice harbouring Sox $4^{\text {flox }}$ were crossed with the Neurog3-Cre transgenic mice (ES4KO). Because reduction of SOX4 expression would likely occur with some delay following Neurog3 induction in the ES4KO mice, we hypothesised that no difference in the number of NEUROG $3^{+}$cells would be observed. To test this, we analysed the number of NEUROG ${ }^{+}$cells in the ES4KO pancreas at E15.5 and found no difference between genotypes (Fig. 5a-c). Despite this, quantification of endocrine cells within E18.5 ES4KO pancreas demonstrated significant reductions of $\mathrm{INS}^{+}, \mathrm{GCG}^{+}$(Fig. $5 \mathrm{~d}-\mathrm{g}$ ), $\mathrm{SST}^{+}$ (Fig. 5h, i, k) and SOX4 $4^{+}$(ESM Fig. 2e) cells but not $\mathrm{GHRL}^{+}$ (Fig. 5h-j) or PPY (Fig. 51-n) cells.

Loss of SOX4 does not change the number of Neurog3 lineage cells Because a significant reduction in endocrine mass was observed in the ES4KO mouse and SOX4 has previously been shown to be important for cell survival and cell proliferation [45], the effects of loss of SOX4 on proliferation (ESM Fig. 6a-c) and apoptosis (ESM Fig. 6d-g) were tested: neither was affected.

Previous studies have suggested 'dedifferentiated' endocrine lineage cells maintain expression of the neuroendocrine secretory granule protein chromogranin A (CHGA) [46]. Therefore, in order to address the possibility that islet endocrine cells do not fully differentiate in ES4KO mice, CHGA immunostaining was carried out. Remarkably, despite the reduction in $\mathrm{INS}^{+}$(Figs 5d, e and 6b) and other endocrine cells (Fig. 5) no difference in the number of $\mathrm{CHGA}^{+}$cells (Fig. 6a) was observed. Furthermore, co-staining for INS and GCG with CHGA uncovered a significantly increased number of cluster-forming (dotted area) CHGA-expressing cells that did not co-stain for either of these hormones (Fig. 6c, d). These data suggest that loss of SOX4 in the ES4KO impacts enriched at the Neurog3 promoter. Luciferase assays demonstrate that SOX 4 activates a truncated $2 \mathrm{~kb}$ fragment of Neurog 3 in mPACL2 0 cells (c). $n \geq 3 ;{ }^{*} p \leq 0.05$. RLU, relative light units

normal islet endocrine cell differentiation or maturation downstream of NEUROG3.

To show that these CHGA-expressing non-INS ${ }^{+} / \mathrm{GCG}^{+}$ cells were derived from the Sox4-null Neurog3 lineage, mice harbouring a $R O S A 26^{\mathrm{mT} / \mathrm{mG}}$ reporter allele [32] were crossed with ES4KO mice (EMS4KO). In this model, Cre expression in endocrine progenitors drives a switch from red fluorescence (RF) to green fluorescence (GF). Neurog3-lineage $\mathrm{GFP}^{+}$cells were quantified and no reduction in the total number of $\mathrm{GFP}^{+}$ cells in the EMS4KO mice was found (Fig. 6e), supporting the observation that neither cell proliferation nor death is impacted. Despite a similar number of pancreatic $\mathrm{GFP}^{+}$cells, a significant increase in non- $\mathrm{INS}^{+} / \mathrm{GCG}^{+}$-staining $\mathrm{GFP}^{+}$cells was observed in the EMS4KO mice (Fig. 6f-h). Notably, no differences in the numbers of $\mathrm{GFP}^{+}$cells that expressed the acinar cell marker amylase (ESM Fig. 7a, b, e) or the ductal cell marker Dolichos biflorus agglutinin (DBA)-lectin (ESM Fig. 7c, d, f) were observed. These experiments reveal that Sox4-knockout cells remain present within the pancreas, incapable of differentiating into islet hormone-expressing endocrine cells and that they persist in a state not normally present in the pancreas, with $\mathrm{CHGA}^{+}$and synaptophysin ${ }^{+}$(not shown) but no islet hormone expression.

SOX4 regulates factors downstream of Neurog3 As lineage tracing revealed that Sox4-null Neurog3 lineage cells remained fated to a neuroendocrine lineage, the possibility that SOX4-regulated genes important for beta cell differentiation downstream of Neurog3 was explored. To that end, qPCR expression analyses were carried out at E17.5 (Fig. 7a-j). The expression profiles of the endocrine hormones (Fig. 7a-e) mirrored tissue morphometry results (Fig. 5). Importantly, gene expression of at least two transcription factors known to be downstream of endocrine cell specification, Neurod1 and Pax4, was significantly reduced (Fig. 7f, g). 
Fig. 5 ES4KO embryos have defective endocrine cell formation. Pancreatic immunostaining for $(\mathbf{a}, \mathbf{b})$ NEUROG3 (green) and SOX9 (red) and subsequent morphometric analyses showed no difference in NEUROG3 ${ }^{+}$(c) or $\mathrm{SOX}^{+}$cells (not shown) in the ES4KO (compared b vs a). Immunostaining in control $(\mathbf{d}, \mathbf{h}$, l) and ES4KO (e, i, m) E18.5 pancreases for: (d, e) $\mathrm{INS}^{+}$(green) and $\mathrm{GCG}^{+}$(red), (h, i) GHRL (green) and $\mathrm{SST}^{+}$(red) and $(\mathbf{l}, \mathbf{m})$ $\mathrm{PPY}^{+}$(green) and $\mathrm{INS}^{+}$(red). Morphometric analyses showed that ES4KO (black bars) have significantly reduced $\mathrm{INS}^{+}$(f), $\mathrm{GCG}^{+}(\mathbf{g})$ and $\mathrm{SST}^{+}(\mathbf{k})$ cell populations but normal $\mathrm{GHRL}^{+}$ (j) and $\mathrm{PPY}^{+}$(n) cell populations compared with controls (white bars). $n \geq 3 ;{ }^{*} p \leq 0.05$; scale bars, $50 \mu \mathrm{m}$; nuclei are stained blue

Fig. 6 Sox4-null endocrine progenitor cells remain in the endocrine lineage but do not differentiate into islet endocrine cells. $\mathrm{CHGA}^{+}$(a), but not $\mathrm{INS}^{+}$ (b), cells are present in normal numbers in the E18.5 ES4KO (black bars). Significant numbers of $\mathrm{CHGA}^{+} \mathrm{INS}^{-} \mathrm{GCG}^{-}$cells can be appreciated in the $\operatorname{ES} 4 \mathrm{KO}$ (d, dotted area) but not in control animals (c, arrowheads). There is no change in the total number of labelled endocrine lineage $\mathrm{GFP}^{+}$ cells in the E18.5 ES4KO pancreas (e). However, significantly increased numbers of $\mathrm{GFP}^{+}$cells that did not co-stain for either INS or GCG were observed (f, $\mathbf{h}$, dotted area) compared with littermate controls (white bars, $\mathbf{g}$, arrowheads). $n \geq 3$; ${ }^{*} p \leq 0.05$; scale bars, $50 \mu \mathrm{m}$; nuclei are stained blue
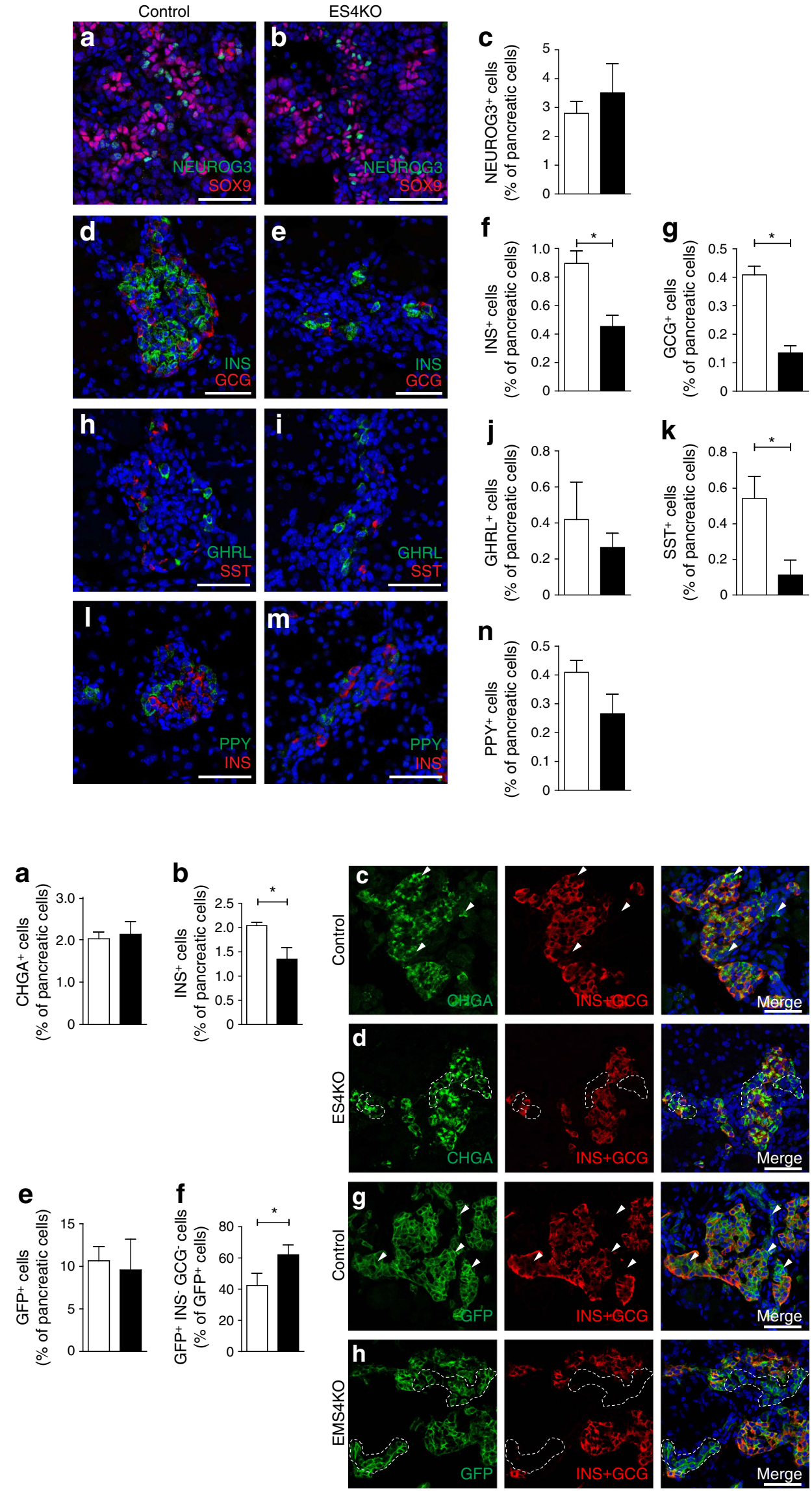
Fig. 7 SOX4 potentiates the induction of pro-beta cell genes during endocrine pancreas development. Ins2 (a), Gcg (b), Sst (d), Ppy (e), Pax4 (f), Neurod1 (g), and Sox4 (h) message levels were significantly reduced, while Ghrl (c), Sox11 (i), and Sox12 (j) were not in E17.5 ES4KO (black bars). Pax4 (k) and Neurodl (l) expression was induced following combined Ad-Neurog3 and Ad-Sox4 in mPACL20 cells. SOX4 significantly induced Neurodl promoter-driven luciferase activity in MPACL20 cells $(\mathbf{m})$. ChIP demonstrated that HA-tagged SOX4 was enriched at the Pax4 and Neurod1 promoters in the Ad-Neurog3-transduced mPAC-Sox4-HA cells $(\mathbf{n} ; n=3)$. qPCR analyses of Sox 4 (o); Neurog3 (p); Neurod1 (q); and Pax4 (r) expression in newborn endocrine cells from E17.5 control and EMS4KO mice. A, Tomato $^{+}$; B, Tomato $^{+} \mathrm{GFP}^{+}$ (newborn); and $\mathrm{C}, \mathrm{GFP}^{+}$ (Neurog3 lineage). $n \geq 3 ;{ }^{*} p \leq 0.05$. RLU, relative light units
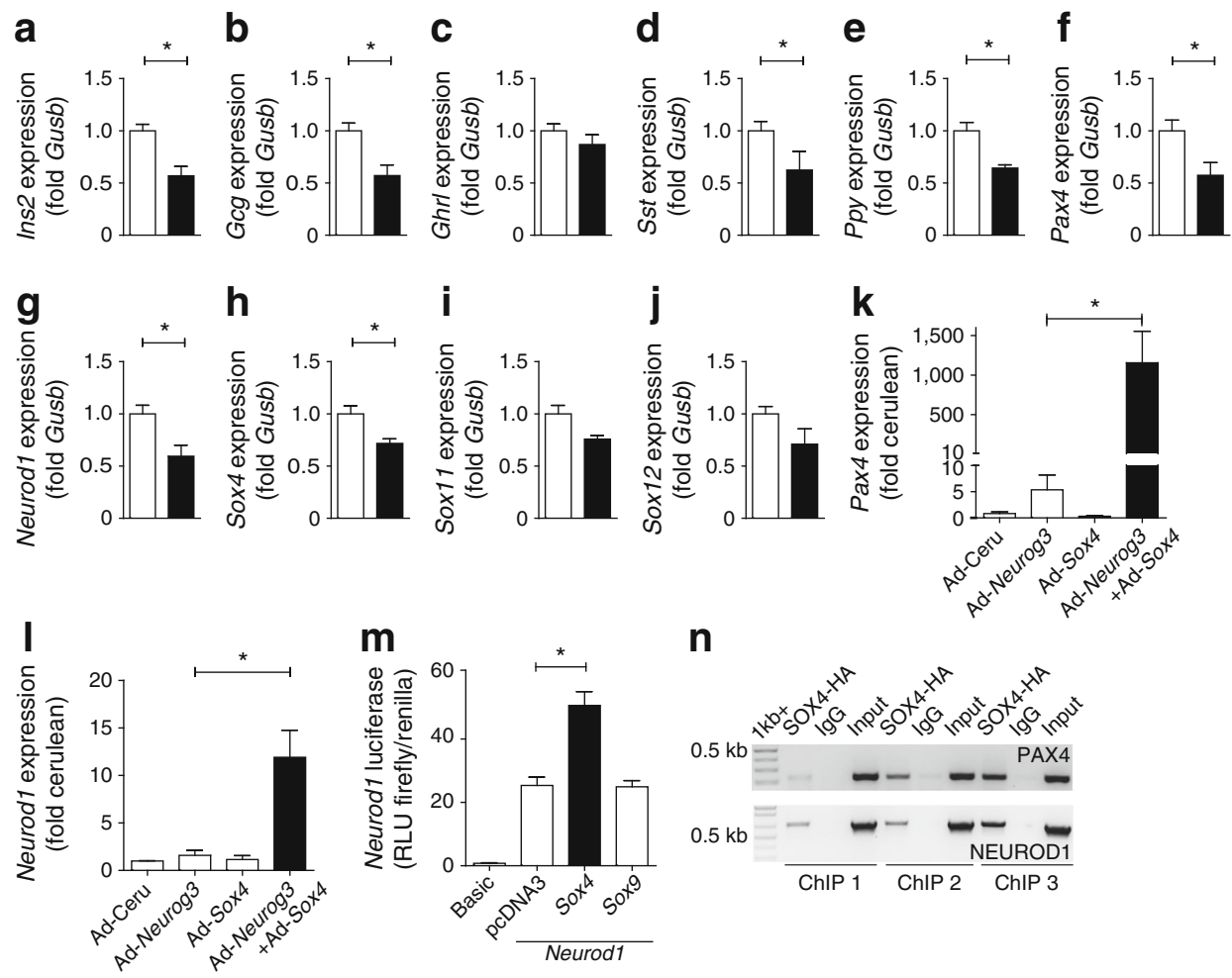

n
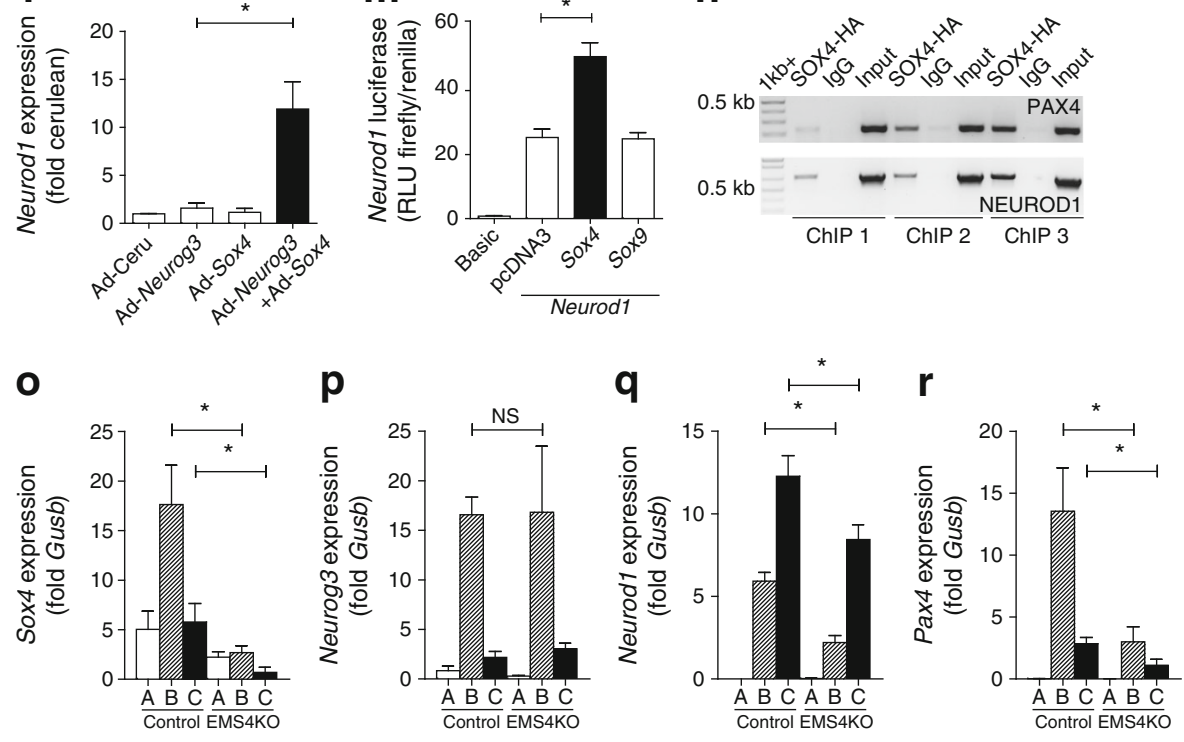

In order to test whether SOX4 binding to the Pax4 and Neurod1 promoters was important for beta cell formation, mPACL20 cells were transduced with Ad-Neurog3 and/or Ad-Sox4, and both Pax4 (Fig. 7k) and Neurodl (Fig. 71) expression was assessed using qPCR. Remarkably, in this system the combination of both Ad-Neurog3 and Ad-Sox4 induced Pax4 expression 200-fold and Neurod 1 expression tenfold compared with Neurog3 alone. Neurod1 promoterdriven luciferase transcription [47] was significantly induced with co-transfection of Sox4 (Fig. 7m). HA-ChIP on Ad-Neurog3-transduced mPAC-Sox4-HA cells demonstrated interaction of SOX4-HA at potential binding sites within both the Pax4 and Neurod1 (Fig. 7n) promoters, providing further evidence for the direct regulation of these beta cell transcription factors by SOX4.

In order to understand if Pax4 and Neurod1 are SOX4 targets in vivo, FACS purification of 'newborn' Neurog3-lineage cells was carried out [32, 48] (Fig. 7o-r; ESM Fig. 8) using the Neurog3-Cre $\mathrm{mT} / \mathrm{mG}$ mouse. We reasoned that newly born cells would possess both RF and GF and FACS purification of these cells demonstrated significant enrichment of both Neurog3 and Sox4, indicating that SOX4 is indeed enriched in the newborn endocrine progenitor pool in vivo (Fig. 7o, p). Assessment by FACS suggests that Neurog3-Cre mediated SOX4 inactivation is robust in the EMS4KO line, with over $80 \%$ loss of Sox 4 message in the endocrine lineage (Fig. 7o). More robust Sox 4 message detected by whole pancreas qPCR (Fig. 7h) may be due to low-level expression in the exocrine tissues, which form the bulk of the pancreas. In agreement with in vitro studies, significant reductions in expression of Pax4, Neurod1 and Ins2 (but not aristaless related homeobox [Arx]) were observed in the Neurog3-lineage cells from the EMS4KO (Fig. 7q, r; ESM Fig. 8c, d). The previous demonstrations that Pax4 and Neurod 1 are directly downstream of Neurog 3 and the observation that expression of these factors was reduced in the newborn $(\mathrm{RF}+\mathrm{GF})$ endocrine cells that expressed normal Neurog3 levels (Fig. 7p), lends credence to them being direct, biologically important, SOX4 targets in vivo. 


\section{Discussion}

Many of the developmental transitions that underlie the formation of beta cells are marked by expression of unique SOX proteins. In embryonic stem cells, SOX2 helps maintain the network of factors that are important for the pluripotent state [49]. SOX factors remain important downstream of SOX2 during definitive endoderm formation, when octamerbinding transcription factor 4 (OCT4) complexes with SOX17 to regulate endoderm induction [50]. SOX17 then likely interacts with undefined factors until expression wanes in the pancreatic buds at the time when SOX9 is induced [51]. SOX9 establishes the gene expression programme necessary for pancreatic budding and growth and remains important in the formation of the endocrine progenitor cells [52]; however, it is silenced in the endocrine lineage.

This study provides the first evidence of a cell-autonomous role for SOX4 during normal endocrine cell formation. Based on our data, we propose a model (Fig. 8) where SOX4 within a subset of multipotent epithelial progenitor cells stabilises Neurog3 transcription and endocrine progenitor fate. SOX4 then couples endocrine progenitor cell differentiation with subsequent development through cooperation with NEUROG3.

SOX4 has been implicated in development of a wide array of mammalian tissues. As such, the previous pancreatic studies did not definitively show that cell-autonomous actions of SOX4 in the pancreatic epithelium were necessary [28, 31, 53]. Furthermore, the early studies did not uncover when SOX4 becomes important for islet cell formation or how it functions to regulate islet cell genesis. Finally, these previous studies did not attempt to assess whether SOX4 expression in the exocrine progenitors is important for their differentiation. Herein, we address all of these previous questions and for the first time show that SOX4 expression within the trunk cells is necessary for endocrine cell development and that loss of SOX4 impacts both formation of progenitors and terminal differentiation of all islet cells but not the exocrine tissue.

Of note, Danio rerio Sox $4 b$, an orthologue of Sox4, was transiently expressed in endocrine precursor cells and knockdown of SOX4B uncovered a requirement for alpha cell differentiation. Other endocrine cell types, however, were unchanged [29]. The differences in observed phenotypes between our study and this former one may be attributed in part to unique endocrine differentiation pathways present in fish and mice or to the differences in knockout/knockdown approaches used.

In the multipotent pancreatic progenitor cells, Neurog3 expression is repressed through active NOTCH signalling $[54,55]$. Endocrine cell differentiation requires loss of NOTCH signalling and binding of a number of transcription factors to the Neurog3 promoter including: one cut homeobox 1 (ONECUT1), SOX9, hepatocyte nuclear factor $1 \beta$ (HNF1B), forkhead box protein A2 (FOXA2) and NEUROG3 itself $[15,42,54,56-58]$. This study adds to the cadre of factors that regulate NEUROG3 by demonstrating that SOX4 participates in this NEUROG3 regulatory network.

SOX4 remains important for endocrine differentiation downstream of its initial role in activating NEUROG3 protein expression. Pax4 and Neurod1, which lie directly downstream of Neurog3 and are essential during beta cell formation [24, 59], were significantly reduced in the ES4KO pancreas. Null mutation of Neurodl leads to reductions in alpha, beta and delta cells [20] while pancreatic Pax4-knockouts have reductions of both beta and delta cells and increases in the other endocrine cells including $\mathrm{GHRL}^{+}$epsilon cells [24, 60, 61]. Notably, a dramatic increase in epsilon cells was not observed following loss of SOX4, suggesting that residual Pax4 expression is sufficient to suppress epsilon cell formation. Interestingly, the highly abundant epsilon cells in the Pax4-null pancreas also express CHGA [60]; thus, the possibility remains that loss of SOX4 drives endocrine differentiation to an unappreciated terminally differentiated non-islet endocrine cell type.

A number of studies have characterised transcription factors and their regulatory networks in pancreatic development. This knowledge has catalysed advancements of directed differentiation protocols and has brought cell-based strategies for the generation of functional INS-producing cells to clinical trials. In conclusion, this work expands the network of transcription factors that are directly important for beta cell formation, and future studies that aim to understand how SOX4 is induced within endocrine progenitors may refine protocols for differentiation of beta cells from hESCs.

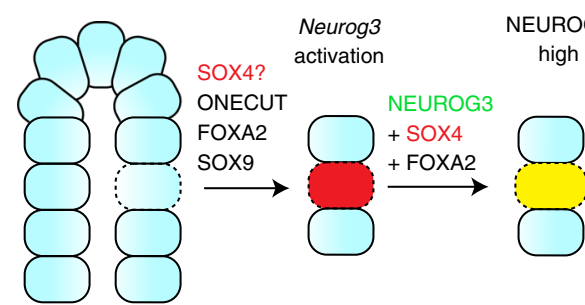

Fig. 8 Model highlighting a role for SOX4 during pancreas development. SOX4-expressing bipotent progenitor cells (red) activate high levels of NEUROG3 expression (yellow). SOX4 and NEUROG3 cooperate following Neurog3 activation to increase NEUROG3 levels and also to increase expression of downstream targets Pax4 and Neurod1, genes necessary for mature endocrine cell formation (light and dark blue) 
Acknowledgements We thank A. Grapin-Botton (University of Copenhagen, Copenhagen, Denmark), M. S. German (University of California San Francisco, San Francisco, CA, USA), S. B. Smith (University of California San Francisco, San Francisco, CA, USA), L. Sussel (Columbia University, New York, NY, USA), D. Melton (Harvard University, Cambridge, MA, USA) and D. Hanahan (École Polytechnique Fédérale de Lausanne, Lausanne, Switzerland) for their generous contributions of reagents that made this work possible. We thank all members of the Lynn laboratory as well as R. Gasa (Institut d'Investigacions Biomèdiques August Pi i Sunyer, Barcelona, Spain) and T. Miyatsuka for their thoughtful reading of the manuscript.

Duality of interest The authors declare that there is no duality of interest associated with this manuscript.

Funding This work was supported by grants to FCL from the JDRF (2-2011-91) and the Canadian Institutes of Health Research (CIHR) (MOP 102628). The Michael Smith Foundation for Health Research, the Canadian Diabetes Association and the Child and Family Research Institute (CFRI) provided salary support for FCL. EEX and NAJK were recipients of CIHR-BC Transplantation Trainee Fellowships and EEX was a recipient of a CFRI graduate studentship.

Contribution statement EEX, NAJK, ST, SZC, MT, CN, and FCL designed, acquired and analysed data. FCL and EEX drafted the manuscript and all authors subsequently revised the manuscript for important intellectual content. All authors approve the version of the manuscript to be published. FCL is the guarantor of the integrity of this work.

\section{References}

1. Seymour PA, Freude KK, Tran MN et al (2007) SOX9 is required for maintenance of the pancreatic progenitor cell pool. Proc Natl Acad Sci U S A 104:1865-1870

2. Kawaguchi Y, Cooper B, Gannon M et al (2002) The role of the transcriptional regulator Ptfla in converting intestinal to pancreatic progenitors. Nat Genet 32:128-134

3. Jonsson J, Carlsson L, Edlund T, Edlund H (1994) Insulin-promoterfactor 1 is required for pancreas development in mice. Nature 371 : 606-609

4. Lammert E, Cleaver O, Melton D (2001) Induction of pancreatic differentiation by signals from blood vessels. Science 294:564-567

5. Kim SK, Melton DA (1998) Pancreas development is promoted by cyclopamine, a hedgehog signaling inhibitor. Proc Natl Acad Sci U S A 95:13036-13041

6. Deutsch G, Jung J, Zheng M et al (2001) A bipotential precursor population for pancreas and liver within the embryonic endoderm. Development 128:871-881

7. Hebrok M, Kim SK, St Jacques B et al (2000) Regulation of pancreas development by hedgehog signaling. Development 127:4905-4913

8. Kim SK, Hebrok M, Li E et al (2000) Activin receptor patterning of foregut organogenesis. Genes Dev 14:1866-1871

9. Pictet RL, Clark WR, Williams RH, Rutter WJ (1972) An ultrastructural analysis of the developing embryonic pancreas. Dev Biol 29: 436-467

10. Shih HP, Wang A, Sander M (2013) Pancreas organogenesis: from lineage determination to morphogenesis. Annu Rev Cell Dev Biol 29:81-105

11. Gouzi M, Kim YH, Katsumoto K et al (2011) Neurogenin3 initiates stepwise delamination of differentiating endocrine cells during pancreas development. Dev Dyn 240:589-604

12. Gradwohl G, Dierich A, LeMeur M, Guillemot F (2000) Neurogenin3 is required for the development of the four endocrine cell lineages of the pancreas. Proc Natl Acad Sci U S A 97:16071611

13. Zhou Q, Law AC, Rajagopal J et al (2007) A multipotent progenitor domain guides pancreatic organogenesis. Dev Cell 13:103-114

14. Kopp JL, Dubois CL, Hao E et al (2011) Progenitor cell domains in the developing and adult pancreas. Cell Cycle 10:1921-1927

15. Jensen J, Pedersen EE, Galante P et al (2000) Control of endodermal endocrine development by Hes-1. Nat Genet 24:36-44

16. Murtaugh LC, Stanger BZ, Kwan KM, Melton DA (2003) Notch signaling controls multiple steps of pancreatic differentiation. Proc Natl Acad Sci U S A 100:14920-14925

17. Gu G, Dubauskaite J, Melton DA (2002) Direct evidence for the pancreatic lineage: NGN3+ cells are islet progenitors and are distinct from duct progenitors. Development 129:2447-2457

18. Miyatsuka T, Li Z, German MS (2009) Chronology of islet differentiation revealed by temporal cell labeling. Diabetes 58:1863-1868

19. Ahlgren U, Pfaff SL, Jessell TM et al (1997) Independent requirement for ISL1 in formation of pancreatic mesenchyme and islet cells. Nature 385:257-260

20. Naya FJ, Huang HP, Qiu Y et al (1997) Diabetes, defective pancreatic morphogenesis, and abnormal enteroendocrine differentiation in BETA2/neuroD-deficient mice. Genes Dev 11:2323-2334

21. Sander M, Neubüser A, Kalamaras J et al (1997) Genetic analysis reveals that PAX6 is required for normal transcription of pancreatic hormone genes and islet development. Genes Dev 11:1662-1673

22. Smith SB, Qu H-Q, Taleb N et al (2010) Rfx6 directs islet formation and insulin production in mice and humans. Nature 463:775-780

23. Sussel L, Kalamaras J, Hartigan-O'Connor DJ et al (1998) Mice lacking the homeodomain transcription factor $\mathrm{Nkx} 2.2$ have diabetes due to arrested differentiation of pancreatic beta cells. Development 125:2213-2221

24. Sosa-Pineda B, Chowdhury K, Torres M et al (1997) The Pax4 gene is essential for differentiation of insulin-producing beta cells in the mammalian pancreas. Nature 386:399-402

25. Matsuoka T, Zhao L, Artner I et al (2003) Members of the large Maf transcription family regulate insulin gene transcription in islet beta cells. Mol Cell Biol 23:6049-6062

26. Wegner M (2010) All purpose Sox: the many roles of Sox proteins in gene expression. Int J Biochem Cell Biol 42:381-390

27. Kamachi Y, Kondoh H (2013) Sox proteins: regulators of cell fate specification and differentiation. Development 140:4129-4144

28. Lioubinski O, Müller M, Wegner M, Sander M (2003) Expression of Sox transcription factors in the developing mouse pancreas. Dev Dyn 227:402-408

29. Mavropoulos A, Devos N, Biemar F et al (2005) Sox4b is a key player of pancreatic alpha cell differentiation in zebrafish. Dev Biol 285:211-223

30. McDonald E, Li J, Krishnamurthy M et al (2012) SOX9 regulates endocrine cell differentiation during human fetal pancreas development. Int J Biochem Cell Biol 44:72-83

31. Wilson ME, Yang KY, Kalousova A et al (2005) The HMG box transcription factor Sox 4 contributes to the development of the endocrine pancreas. Diabetes 54:3402-3409

32. Muzumdar MD, Tasic B, Miyamichi K et al (2007) A global doublefluorescent Cre reporter mouse. Genesis 45:593-605

33. Schonhoff SE, Giel-Moloney M, Leiter AB (2004) Neurogenin 3 -expressing progenitor cells in the gastrointestinal tract differentiate into both endocrine and non-endocrine cell types. Dev Biol 270:443-454

34. Penzo-Méndez A, Dy P, Pallavi B, Lefebvre V (2007) Generation of mice harboring a Sox 4 conditional null allele. 780:776-780

35. Sabatini PV, Krentz NAJ, Zarrouki B et al (2013) Npas4 is a novel activity-regulated cytoprotective factor in pancreatic $\beta$-cells. Diabetes 62:2808-2820 
36. Carpenter AE, Jones TR, Lamprecht MR et al (2006) Cell profiler: image analysis software for identifying and quantifying cell phenotypes. Genome Biol 7:R100

37. Luo J, Deng Z-L, Luo X et al (2007) A protocol for rapid generation of recombinant adenoviruses using the AdEasy system. Nat Protoc 2: 1236-1247

38. Seymour PA, Freude KK, Dubois CL et al (2008) A dosagedependent requirement for Sox9 in pancreatic endocrine cell formation. Dev Biol 323:19-30

39. Schaffer AE, Freude KK, Nelson SB, Sander M (2010) Nkx6 transcription factors and Ptfla function as antagonistic lineage determinants in multipotent pancreatic progenitors. Dev Cell 18:1022-1029

40. Yoshida T, Hanahan D (1994) Murine pancreatic ductal adenocarcinoma produced by in vitro transduction of polyoma middle T oncogene into the islets of Langerhans. Am J Pathol 145:671-684

41. Gasa R, Mrejen C, Leachman N et al (2004) Proendocrine genes coordinate the pancreatic islet differentiation program in vitro. Proc Natl Acad Sci U S A 101:13245-13250

42. Lynn FC, Smith SB, Wilson ME et al (2007) Sox9 coordinates a transcriptional network in pancreatic progenitor cells. Proc Natl Acad Sci U S A 104:10500-10505

43. Lee JC, Smith SB, Watada H et al (2001) Regulation of the pancreatic pro-endocrine gene neurogenin3. Diabetes 50:928-936

44. Rukstalis JM, Habener JF (2009) Neurogenin3: a master regulator of pancreatic islet differentiation and regeneration. Islets 1:177-184

45. Bhattaram P, Penzo-Méndez A, Sock E et al (2010) Organogenesis relies on SoxC transcription factors for the survival of neural and mesenchymal progenitors. Nat Commun 1:9

46. Talchai C, Xuan S, Kitamura T et al (2012) Generation of functional insulin-producing cells in the gut by Foxo1 ablation. Nat Genet 44: 406-412

47. Anderson KR, Torres CA, Solomon K et al (2009) Cooperative transcriptional regulation of the essential pancreatic islet gene NeuroD1 (beta2) by Nkx2.2 and neurogenin 3. J Biol Chem 284:31236-31248

48. Xiao X, Chen Z, Shiota C et al (2013) No evidence for $\beta$ cell neogenesis in murine adult pancreas. J Clin Invest 123:2207-2217
49. Boyer LA, Lee TI, Cole MF et al (2005) Core transcriptional regulatory circuitry in human embryonic stem cells. Cell 122:947-956

50. Aksoy I, Jauch R, Chen J et al (2013) Oct4 switches partnering from Sox 2 to Sox 17 to reinterpret the enhancer code and specify endoderm. EMBO J 32:938-953

51. Choi E, Kraus MRC, Lemaire LA et al (2012) Dual lineage-specific expression of Sox 17 during mouse embryogenesis. Stem Cells 30: 2297-2308

52. Lynn FC, Skewes-Cox P, Kosaka Y et al (2007) MicroRNA expression is required for pancreatic islet cell genesis in the mouse. Diabetes 56:2938-2945

53. Goldsworthy M, Hugill A, Freeman H, Horner E (2008) Role of the transcription factor sox 4 in insulin secretion and impaired glucose tolerance. Diabetes 57:2234-2244

54. Apelqvist A, Li H, Sommer L et al (1999) Notch signalling controls pancreatic cell differentiation. Nature 400:877-881

55. Qu X, Afelik S, Jensen JN et al (2013) Notch-mediated post-translational control of Ngn3 protein stability regulates pancreatic patterning and cell fate commitment. Dev Biol 1-12

56. Schwitzgebel VM, Scheel DW, Conners JR et al (2000) Expression of neurogenin3 reveals an islet cell precursor population in the pancreas. Development 127:3533-3542

57. Wang S, Jensen JN, Seymour PA et al (2009) Sustained Neurog3 expression in hormone-expressing islet cells is required for endocrine maturation and function. Proc Natl Acad Sci U S A 106:9715-9720

58. Lee C, Sund N, Vatamaniuk M (2002) Foxa2 controls Pdx1 gene expression in pancreatic beta cells in vivo. Diabetes 51:2546-2551

59. Smith SB, Gasa R, Watada H et al (2003) Neurogenin3 and hepatic nuclear factor 1 cooperate in activating pancreatic expression of Pax4. J Biol Chem 278:38254-38259

60. Prado CL, Pugh-Bernard AE, Elghazi L et al (2004) Ghrelin cells replace insulin-producing beta cells in two mouse models of pancreas development. Proc Natl Acad Sci U S A 101:2924-2929

61. Wang Q, Elghazi L, Martin S et al (2008) Ghrelin is a novel target of Pax4 in endocrine progenitors of the pancreas and duodenum. Dev Dyn 237:51-61 